\title{
Effects of substituent position on the interactions between PBDEs/PCBs and DOM
}

\author{
NUERLA AiLiJiang, QIAO XianLiang*, LI Jing, ZHAO DongMei, YANG XianHai, \\ XIE Qing \& CHEN JingWen
}

Key Laboratory of Industrial Ecology and Environmental Engineering of Ministry of Chemistry, School of Environmental Science and Technology, Dalian University of Technology, Dalian 116024, China

Received March 23, 2012; accepted May 17, 2012; published online September 20, 2012

\begin{abstract}
Dissolved organic matters (DOM) have important influence on the environmental behavior and fate of organic pollutants, therefore rationalization of interaction mechanisms between pollutants and DOM has become a hot topic in the field of environmental studies. In this paper, polybrominated diphenyl ethers (PBDEs) and polychlorinated biphenyls (PCBs), widely detected pollutants, were chosen as target compounds. The effects of substituent position on the interaction between PBDEs/PCBs and DOM were explored. Equilibrium dialysis technique combined with quantum chemistry and molecular docking calculations were employed to reveal the interaction mechanism from the view of charge distribution and molecular conformation. It is shown that non-ortho-substituted isomers have larger volumes and stronger hydrophobicity than those of ortho-substituted, therefore non-ortho-substituted isomers bind more favorably with DOM by hydrophobic partition. By calculating the atomic charge distribution of target chemicals and Leonardite humic acid (LHA) molecular model, $\pi-\pi$ interactions between the aromatic rings of target chemicals with LHA are proposed and further proved in molecular docking calculations. There were 10, 8, 6 docking conformations demonstrating $\pi-\pi$ interaction with LHA for CB-77, BDE-77 and BDE-47, respectively, but none was found for CB-47. By comparing the change of dihedral angle of the aromatic rings and energy barrier, non-ortho-substituted PBDEs/PCBs have larger dihedral angle adjustment space and flexibility, which results in stronger interaction and binding capability with DOM than ortho-substituted isomers. This paper shed some lights on the effect of substituent position on the environmental behaviors of PBDEs and PCBs.
\end{abstract}

dissolved organic matter, polybrominated diphenyl ethers, polychlorinated biphenyls, substituent position, dihedral angle, $\pi-\pi$ interaction

Citation: Nuerla A L J, Qiao X L, Li J, et al. Effects of substituent position on the interactions between PBDEs/PCBs and DOM. Chin Sci Bull, 2013, 58: 884-889, doi: 10.1007/s11434-012-5464-9

The environmental behavior, ecological effects and pollution control of the persistent organic pollutants (POPs) are currently one of important worldwide environmental issues [1-4]. Plybrominated diphenyl ethers (PBDEs), widely used as additive flame retardants, and polychlorinated biphenyls (PCBs), mainly used as dielectric and coolant fluids, are typical POPs. In recent years, PBDEs and PCBs have been released into the environment through various means and detected in diverse environmental media (such as air, water,

*Corresponding author (email: xlqiao@dlut.edu.cn) soil and sediment) and biota samples [1,2]. There has been a growing scientific and societal concern about their environmental behavior and toxicological effects. With high octanol-water partitioning coefficient $\left(K_{\mathrm{OW}}\right)$ values $[5,6]$, PBDEs and PCBs favorably partition to well-defined hydrophobic phase in environment, such as organic matters in soils and sediments. The partition behavior of pollutants in environment were not only influenced by their structures and intrinsic properties, but also were significantly affected by some environmental factors. As one of the most widely distributed ubiquitous natural materials in environment, 
many studies have reported that dissolved organic matter (DOM) can interact with hydrophobic organic pollutants (PAHs, PCBs, DDT, PBDEs, etc.) through hydrophobic partition or electrostatic effects (hydrogen bond or $\pi-\pi$ bond), which would affect the adsorption, transport, bioavailability and other environmental behaviors of these compounds [7-9].

The binding interactions between organic contaminants and DOM depend on the source and nature of DOM [10,11], as well as the hydrophobicity and steric configuration of compounds [12,13]. Cornelissen et al. [14] found that the nonplanar 2,2'-CB absorbed much less to black carbon than planar compounds like anthracene, phenanthrene and 4-CB. Using equilibrium dialysis method, Qiao et al. [15] determined the binding constants of different isomers of trenbolone with two commercial humic acids, and found that the binding ability of $\beta$-isomer was significantly higher than that of $\alpha$-isomer. For aromatic compounds, substituent groups make great impact on the distribution characteristics of atomic charges on the benzene ring, and further influence the interaction between molecules, such as $\pi-\pi$ interaction $[13,16]$. Uhle et al. [13] reported that compared to non-ortho-substituted PCBs with the same number of chlorine substituent, the binding affinity of ortho-substituted PCBs was significantly weaker. They also speculated that owing to chlorine substituent in ortho-position, free rotation around carboncarbon was inhibited, which could result in the less effectively interaction with fulvic acid substrate. However, there is no experimental support for this presumption in their study. In fact, it is challenging to reveal and characterize the interaction mechanisms between DOM and pollutants using experimental approaches currently. Instead, molecular simulation techniques are very helpful to study the molecular interactions [17].

In this paper, equilibrium dialysis approach was employed to determine the binding constants between DOM and two PBDEs comprised of different substituent positions, and the binding constants between two PCBs with different substituent positions and LHA were derived from references. Quantum chemistry calculations were applied to characterize the parameters of molecular structure and charge distribution of the target compounds, and molecular docking calculations were employed to reveal the effects of substituent position on the interaction mechanisms between PBDEs/PCBs and DOM. This study will be valuable for the assessment of the behavior and fate of organic pollutants with different halogenated substituents.

\section{Experimental}

\subsection{Chemicals and reagents}

Hexane, dichloromethane and acetonitrile (GC grade) were purchased from TEDIA Firm (USA). Potassium dihydrogen phosphate $\left(\mathrm{KH}_{2} \mathrm{PO}_{4}\right)$ and potassium hydroxide $(\mathrm{KOH})$ were analytical reagent grade from Tianjin Bodi Chemical Company (China). Leonardite humic acid (LHA) was purchased from the International Humic Substances Society, and dialysis bags (Dialysis Membrane 2000 Daltons) were purchased from Spectra Laboratories firm. BDE-47, BDE-77 and F-BDE-47 were purchased from AccuStandard firm (USA).

\subsection{Equilibrium dialysis experiment and sample analysis}

Equilibrium dialysis technique was employed to determine the binding constants of two PBDEs comprised of different substituent positions with representative humic acid (LHA). This method is widely used to measure the binding ability of compounds with DOM, because of the reliability of test results [8]. Dialysis bags filled with $3 \mathrm{~mL} 200 \mathrm{mg} / \mathrm{L}$ humic acid were placed in the glass tubes containing solution with the target compound $(2 \mu \mathrm{g} / \mathrm{L})$ buffered with $\mathrm{KH}_{2} \mathrm{PO}_{4}$. After an equilibration period of $48 \mathrm{~h}$, samples of the solutions inside and outside within the dialysis bag were extracted with dichloromethane, and dichloromethane was volatilized and transformed into hexane. Final samples were analyzed with GC-MS.

DB-XLB was used as capillary column $(30.0 \mathrm{~m} \times 250 \mu \mathrm{m}$ $\times 0.1 \mu \mathrm{m})$. Samples were injected to GC in splitless mode. The injector temperature is $290^{\circ} \mathrm{C}$. The temperature program of the $\mathrm{GC}$ oven was as follows: initial temperature $90^{\circ} \mathrm{C}$ for $1 \mathrm{~min}$, then at $30^{\circ} \mathrm{C} / \mathrm{min}$ to $200^{\circ} \mathrm{C}$ (held for $0 \mathrm{~min}$ ), then at $2.5^{\circ} \mathrm{C} / \mathrm{min}$ to $305^{\circ} \mathrm{C}$ (held for $2 \mathrm{~min}$ ), and finally at $5{ }^{\circ} \mathrm{C} / \mathrm{min}$ to $315^{\circ} \mathrm{C}$ (held for $5 \mathrm{~min}$ ). Helium was used as carrier gas at $30 \mathrm{~mL} / \mathrm{min}$. BDE-47, BDE-77 were determined using BDE-28 and F-BDE-47 as recovery substitute and the internal standard respectively. The recoveries for the test procedure were higher than $90 \%$, and the detection limit was $0.1 \mu \mathrm{g} / \mathrm{L}$ for the compound tested.

The binding constant $\left(K_{\mathrm{DOC}}\right)$ is used to characterize the association affinity of PBDEs with DOM, $K_{\mathrm{DOC}}$ was calculated as

$$
K_{\mathrm{DOC}}=\frac{C_{\mathrm{in}}-C_{\mathrm{out}}}{C_{\mathrm{out}} \cdot[\mathrm{DOC}]},
$$

where [DOC] is the concentration of DOM determined by TOC-VCSH analyzer and expressed in $\mathrm{kg} / \mathrm{L}, C_{\text {in }}$ and $C_{\text {out }}$ is the PBDEs concentrations in inside and outside of the dialysis bags respectively, and expressed in $\mu \mathrm{g} / \mathrm{L}, K_{\mathrm{DOC}}$ is expressed in $\mathrm{L} / \mathrm{kg}$.

\subsection{Computation of the molecular structure parameter and charge distribution}

The initial geometries of PBDEs, PCBs and LHA model were firstly optimized by PM3 method using the Chem3D software and then by density functional theory at the B3LYP/ $6-311+G(d, p)$ level. The molecular model of LHA was 
suggested by Niederer et al. [18] according to the data derived from ${ }^{13} \mathrm{C} \mathrm{NMR}$ and elemental analysis. Frequency analysis was performed on the optimized geometries of LHA and compounds to ensure that the system has no imaginary vibration frequencies using the Gaussian 09 program [19]. The IEFPCM model was used to simulate the solvent (water) effects. Based on the optimized structures, the structural descriptors including the balance parameter of surface potential and van der Waals molecular volume were calculated. Finally the optimized structures were also used for natural bond orbital (NBO) analysis at the B3LYP/ $6-311+G(d, p)$ level to calculate the electron distribution of target compounds.

\subsection{Molecular docking}

The CDOCKER protocol incorporated into Discovery Studio 2.5.5 (Accelrys Software Inc., San Diego, CA) was used to carry out the molecular docking. The optimized geometries of PCBs, PBDEs and LHA were used in molecular docking. The CHARMM force field and the default values of all other parameters were used. In the docking process, PBDEs and PCBs molecules are allowed to flex, and the LHA molecule is held rigid. In CDOCKER, random ligand conformations were generated from the initial ligand structure through high-temperature molecular dynamics followed by random rotations. After that, the random conformations were optimized by grid-based simulated annealing, and then the interaction energies between the ligands and receptors were calculated, finally the conformations of complexes were arranged according to the interaction energies. Then binding modes with the lowest interaction energies were obtained as stable modes.

\section{Results and discussion}

\section{1 $\log K_{\mathrm{OW}}$ and $\log K_{\mathrm{DOC}}$}

In this study, we determined the $\log K_{\mathrm{DOC}}$ of $\mathrm{BDE}-47$ and BDE-77 with LHA using equilibrium dialysis. The $\log K_{\text {DOC }}$ of PCBs with HA were derived from ref. [20] and listed in Table 1.

It was shown that the binding constants $\left(\log K_{\mathrm{DOC}}\right)$ of the non-ortho-substituted PBDEs and PCBs with the same humic acid were significantly higher than those of ortho-substituents, and this trend was in correspond with $\log K_{\mathrm{OW}}$, indicating that the binding affinity of the compounds with DOM has good relationship with hydrophobicity. Previous studies have reported that the interactions between molecules not only related to the hydrophobicity of compounds, but also related to the van der Waals forces, hydrogen bonding and $\pi$ electron [21,22]. To reveal the difference between the binding affinities of ortho- and non-ortho-substituted compounds with HA, some molecular structure descriptors of target compounds such as the balance parameter of surface potential $(\tau)$ and van der Waals molecular volume $\left(V_{\mathrm{cm}}\right)$ were calculated. $V_{\mathrm{cm}}$ was used to characterize the size of molecule, and $\tau$ was used to represent the balance degree of the atomic charges, the closer the value of 0.25 , indicating that the better balanced distribution of molecular charge [23]. It was reported that these parameters can reflect the integrity of target molecules and be related to the adsorption and distribution of compounds [15,24]. From the data in Table 1, it can be observed that compared with ortho-substituted CB-47, non-ortho-substituted CB-77 has better balanced distribution of molecular charge, however, non-orthosubstituted BDE-77 shows the opposite trend. The difference of charge distribution between PCBs and PBDEs maybe relate to the $\mathrm{O}$ atom on the ether bond of PBDEs. In addition, the non-ortho-substituted PBDEs/PCBs have larger molecule volumes than ortho-substituted isomers, which indicated larger cavity and stronger repulsion from aqueous phase. Therefore non-ortho-substituted isomers interact more effectively with DOM.

\subsection{Atomic charge distribution}

Previous studies have found that the $\pi-\pi$ interactions are tending to occur between the aromatic compounds comprised of stronger electron donor and accepter substitutes respectively [16,25]. The atomic charge distribution of PBDEs, PCBs, and LHA were obtained and demonstrated in Figure 1 and Table 2 to test the possibility of $\pi-\pi$ interactions between PBDEs/PCBs and DOM. Compared with $\mathrm{Br}$ atoms, $\mathrm{Cl}$ atoms possess greater electron withdrawing ability, which result in larger reduction on the net charge of linked $\mathrm{C}$ atoms. However, the $\mathrm{O}$ atom on the ether bond of PBDEs

Table $1 \log K_{\mathrm{OW}}$, molecular structure parameters and $\log K_{\mathrm{DOC}}$ of the target compounds

\begin{tabular}{|c|c|c|c|c|c|c|}
\hline Compounds & Substituent position & $\log K_{\text {OW }}$ & $V_{\mathrm{cm}}\left(\mathrm{cm}^{3} / \mathrm{mol}\right)$ & $\tau$ & $\log K_{\mathrm{DOC}}$ & $\mathrm{DOM}^{\mathrm{e})}$ \\
\hline BDE-47 & $2,2^{\prime}, 4,4^{\prime}-$ & $7.16^{\text {a) }}$ & 198.5 & 0.188 & $5.80^{\mathrm{c})}$ & LHA \\
\hline BDE-77 & $3,3^{\prime}, 4,4^{\prime}-$ & $7.30^{\text {a) }}$ & 207.6 & 0.174 & $6.21^{\mathrm{c})}$ & LHA \\
\hline CB-47 & $2,2^{\prime}, 4,4^{\prime}-$ & $5.85^{\text {b) }}$ & 172.0 & 0.130 & $4.15^{\mathrm{d})}$ & SW \\
\hline CB-77 & $3,3^{\prime}, 4,4^{\prime}-$ & $6.36^{\mathrm{b})}$ & 177.4 & 0.166 & $5.00^{\mathrm{d})}$ & SW \\
\hline
\end{tabular}

a) Calculated from Sparc on-line calculator http://ibmlc2.chem.uga.edu/sparc/. b) Data from Hawker et al. [6]. c) Data determined in this study. d) Data from Kukkonen et al. [20]. e) LHA is the Leonardite humic acid derived from the brown coal, and SW is the natural organic matter derived from surface water. 


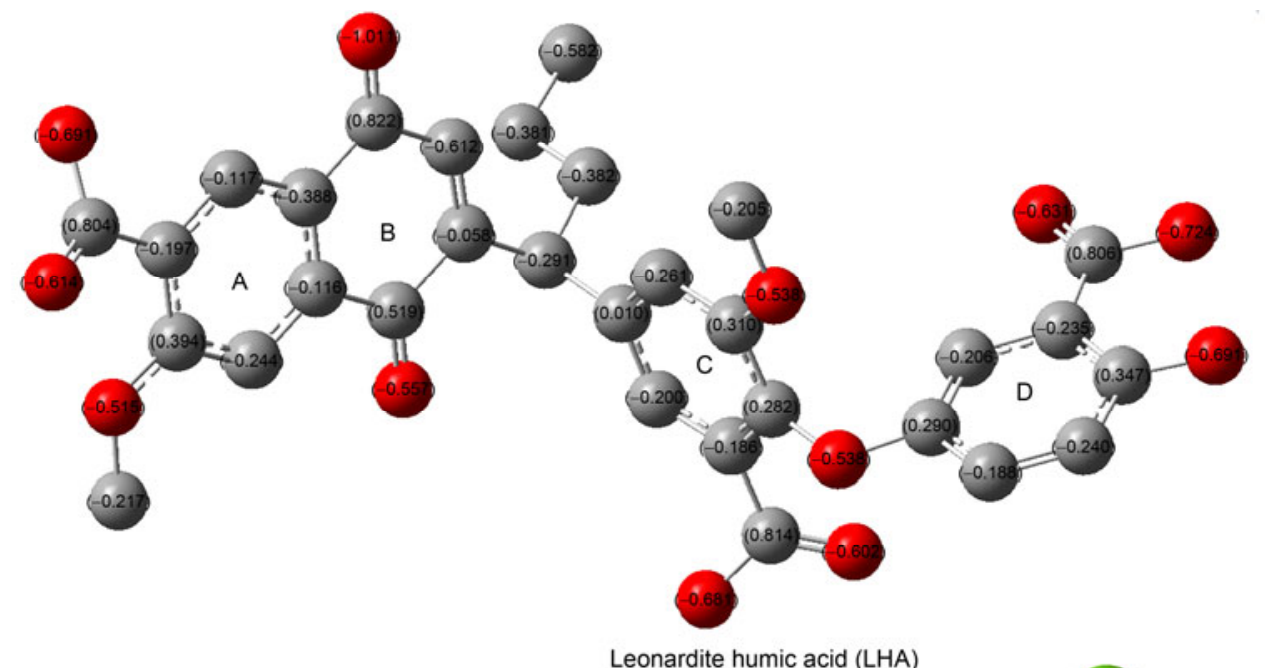

Leonardite humic acid (LHA)

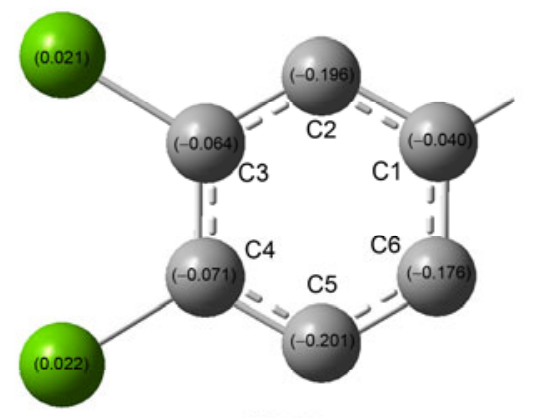

CB-77

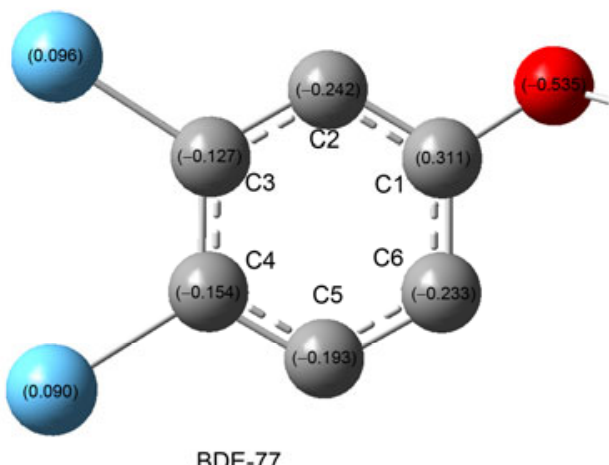

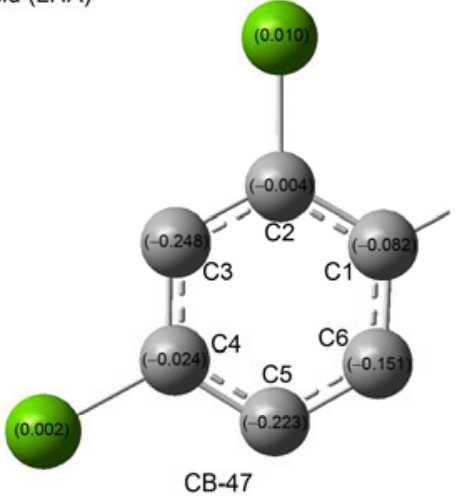

CB-47

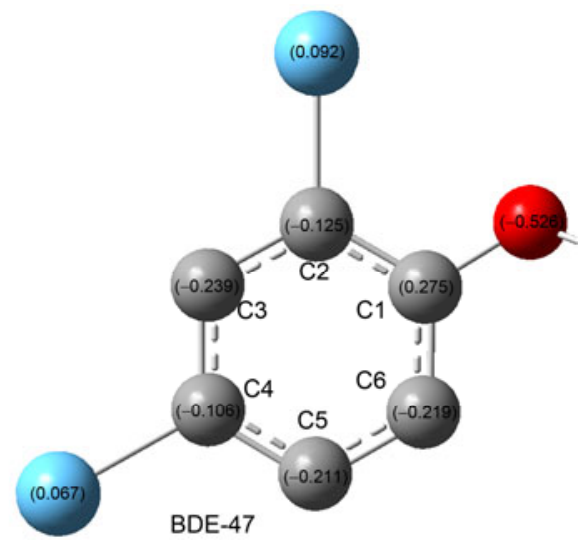

Figure 1 Atomic charge distribution of LHA, CB-47, CB-77, BDE-47 and BDE-77. The balls in red, green, blue, and gray represent oxygen, chlorine, bromine, and carbon atoms, respectively.

has stronger electron withdrawing ability than $\mathrm{Cl}$ atom, which leads to significant decrease in the charge of $\mathrm{C}$ atom connected (Table 2). Generally, benzene rings of both PBDEs and PCBs homologs have negative charge distribution, which might work as donors for $\pi-\pi$ interactions. The LHA model comprised of four benzene rings, named as A, B, C and $\mathrm{D}$ ring respectively. Because of the existence of strong electron-withdrawing function groups (including $-\mathrm{OH}$, $-\mathrm{COOH},-\mathrm{C}=\mathrm{O}$, etc.) on the aromatic rings of LHA model, significant net charge changes are found for $\mathrm{C}$ atoms on the rings, and rather positive net charge were shown on some $\mathrm{C}$ atoms. Therefore, from the view of charge difference, $\pi-\pi$ interactions were highly expected between aromatic rings of PBDEs/PCBs and LHA, which act as electron donor and electron receptor respectively.

\subsection{Molecular docking}

To further investigate how the substituent position affects the binding interactions between PBDEs/PCBs and DOM 
Table 2 The atomic charges of benzene ring of PBDEs, PCBs and LHA

\begin{tabular}{cccccrrrrr}
\hline \multirow{2}{*}{ C atom in molecule } & BDE-47 & \multirow{2}{*}{ BDE-77 } & CB-47 & CB-77 & \multicolumn{4}{c}{ LHA model } \\
\cline { 6 - 9 } & & & & & A ring & B ring & C ring & D ring \\
\hline C1 & 0.275 & 0.311 & -0.082 & -0.040 & 0.394 & 0.519 & 0.310 & -0.188 \\
C2 & -0.125 & -0.242 & -0.004 & -0.196 & -0.244 & -0.058 & 0.282 & -0.240 \\
C3 & -0.239 & -0.127 & -0.248 & -0.064 & -0.116 & -0.612 & -0.186 & 0.347 \\
C4 & -0.106 & -0.154 & -0.024 & -0.071 & -0.388 & 0.822 & -0.200 & -0.235 \\
C5 & -0.211 & -0.193 & -0.223 & -0.201 & -0.117 & -0.388 & 0.010 & -0.206 \\
C6 & -0.219 & -0.233 & -0.151 & -0.176 & -0.197 & -0.116 & -0.261 & 0.290 \\
\hline
\end{tabular}

from the view of steric conformations, molecular docking studies were conducted. The stable binding sites of each target compound with LHA were acquired through molecular docking, and there are 10, 8, 6 conformations showing $\pi-\pi$ interactions for $\mathrm{CB}-77, \mathrm{BDE}-77$ and BDE-47 respectively (Figure 2). Under the same docking parameters, the stable binding mode of CB-47 with LHA could not be found.

Previous studies proposed that dihedral angle was one of the dominant parameters affecting the interactions between PCBs and DOM [13]. The dihedral angles of PBDEs and PCBs before and after docking were calculated. Before docking, the dihedral angle of the two benzene rings in CB-47 and CB-77 are $80.83^{\circ}$ and $141.41^{\circ}$, respectively, and the dihedral angle of BDE-47 and BDE-77 are 107.34 ${ }^{\circ}$ and $108.75^{\circ}$, respectively. In the docking modes, CB-77 has the maximum change range of dihedral angle $\left(34.48^{\circ}-141.42^{\circ}\right)$, revealing the molecule can easily twist and fold, thereby making the molecule interact effectively with LHA. The adjustment ranges of dihedral angle for BDE-77 and BDE47 are $64.56^{\circ}-108.75^{\circ}$ and $91.05^{\circ}-107.34^{\circ}$, respectively. To some extent, the adjustment ranges of dihedral angle between the two benzene rings seem to be one of the main factors to affect the binding of PCBs or PBDEs with DOM.
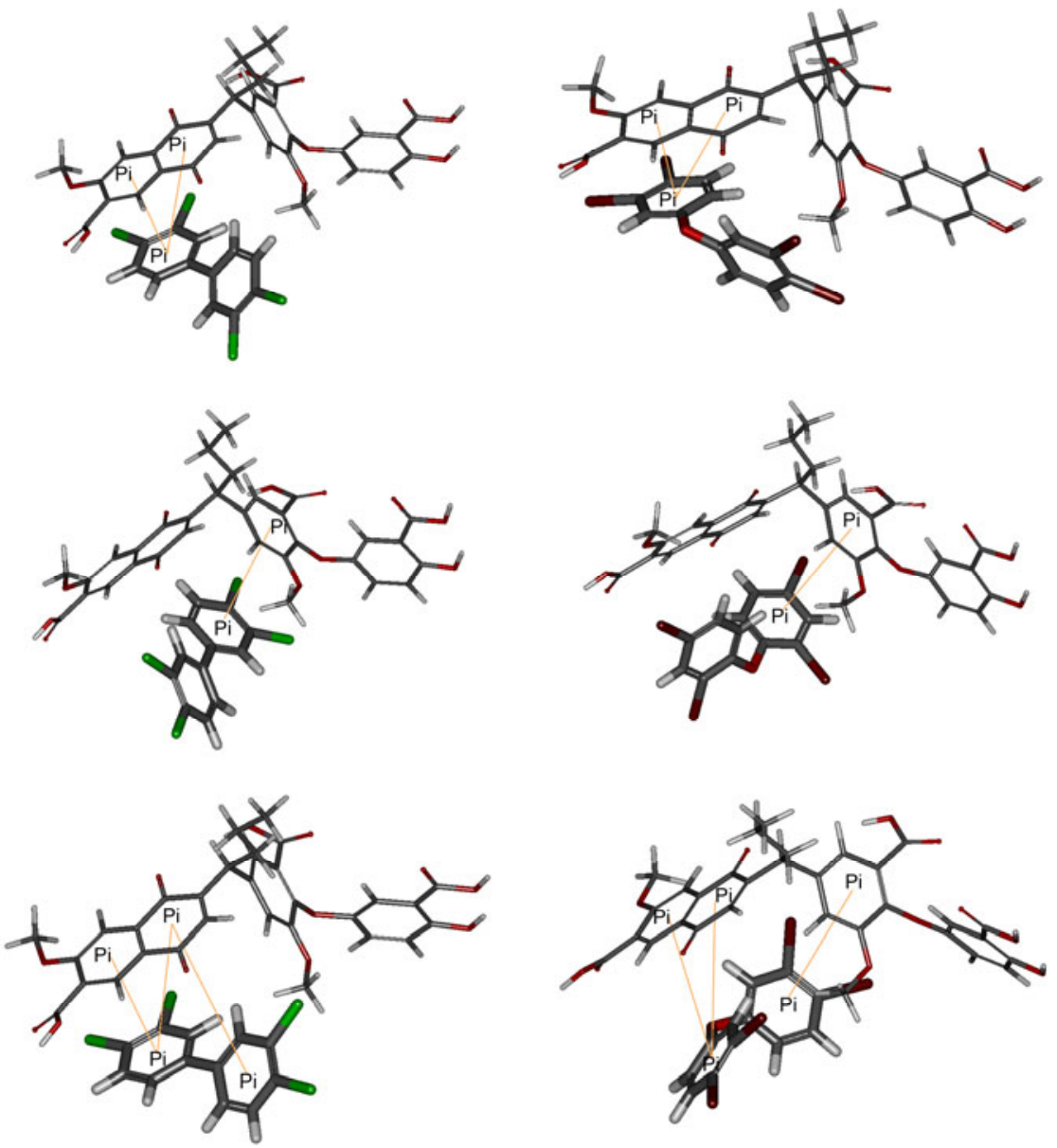

Figure 2 Main binding modes of CB-77 (left) and BDE-77 (right) with LHA in molecular docking. 
In addition, it was also found that if orientation vdw energy threshold of the docking parameters adjusted from 300 to 400 , the stable binding modes of CB-47 with LHA can also be obtained, which indicated that the rotation or twist of ortho-substituted PCB needs more energy to find the stable binding site and conformation with DOM. Though, the LHA model used in this study is not the best represent for diverse DOM in environment, it can provide us with some insights on the binding interactions between organic pollutants and DOM.

\section{Conclusions}

The effects of substituent positions on the interaction between organic pollutants and DOM have been mentioned in previous studies. However, there are short of experimental data to explain the mechanism of substituents induced difference, since it is a challenge to reveal and characterize the interaction mechanisms between DOM and pollutants using experimental approaches. Using equilibrium dialysis experiment, quantum chemical and molecular docking calculations, it was found that substituent positions had important influence on the binding affinities of organic pollutants with DOM. Compared with the ortho-substitutes isomers, nonortho-substituted PBDEs/PCBs have larger molecular volume and stronger hydrophobicity. Moreover, the dihedral angle of the two aromatic rings for non-ortho-substituted PCBs or PBDEs demonstrate larger rotation space and flexibility, thereby interact more effectively with Leonardite humic acid (such as $\pi-\pi$ interactions). With the aid of quantum chemical and molecular docking calculations, our studies give some insight into the influence of halogen atomic substituent position on the interaction between aromatic compounds with DOM. These findings will be helpful in the assessment of environmental behavior and ecological risk of PBDEs/PCBs.

This work was supported by the National Natural Science Foundation of China (21137001, 21077016) and the National High Technology Research and Development Program of China (2012AA06A301).

1 Cynthia A, De W. An overview of brominated flame retardants in the environment. Chemosphere, 2002, 46: 583-624

2 Meijer S N, Ockenden W A, Sweetman A, et al. Global distribution and budget of PCBs and HCB in background surface soils: Implications for sources and environmental processes. Environ Sci Technol, 2003, 37: 667-672

3 Niu J F, Shen Z Y, Yang Z F, et al. Quantitative structure-property relationships on photodegradation of polybrominated diphenyl ethers. Chemosphere, 2006, 64: 658-665

4 Lin H, Niu J F, Ding S Y, et al. Electrochemical degradation of perfluorooctanoic acid (PFOA) by $\mathrm{Ti} / \mathrm{SnO}_{2}-\mathrm{Sb}, \mathrm{Ti} / \mathrm{SnO}_{2}-\mathrm{Sb} / \mathrm{PbO}_{2}$ and
$\mathrm{Ti} / \mathrm{SnO}_{2}-\mathrm{Sb} / \mathrm{MnO}_{2}$ anodes. Water Res, 2012, 46: 2281-2289

5 Braekevelt E, Tittlemier S A, Tomy G T. Direct measurement of octanolwater partition coefficients of some environmentally relevant brominated diphenyl ether congeners. Chemosphere, 2003, 51: 563-567

6 Hawker D W, Connell D W. Octanol-water partition coefficient of polychlorinated biphenyl congener. Environ Sci Technol, 1988, 22: 382-297

7 Chiou C T, Malcolm R L, Brinton T I, et al. Water solubility enhancement of some organic pollutants and pesticides by dissolved humic and fulvic acids. Environ Sci Technol, 1986, 20: 502-508

8 McCarthy J F, Jimenez B D. Interactions between polycyclic aromatic hydrocarbons and dissolved Humic Material: Binding and dissociation. Environ Sci Technol, 1985, 11: 1072-1076

9 Wang W, Moreno L D, Ye Q F, et al. Improved measurements of partition coefficients for polybrominated diphenyl ethers. Environ Sci Technol, 2011, 45: 1521-1527

10 Chin Y P, Aiken G R, Danielsen K M. Binding of pyrene to aquatic and commercial humic substances: The role of molecular weight and aromaticity. Environ Sci Technol, 1997, 31: 1630-1635

11 Kang S, Xing B S. Phenanthrene sorption to sequentially extracted soil humic acids and humans. Environ Sci Technol, 2005, 39: 134-140

12 Burkhard L P. Estimating dissolved organic carbon partition coefficients for nonionic organic chemicals. Environ Sci Technol, 2000, 34: 4663-4668

13 Uhle M E, Chin Y, Aiken G R, et al. Binding of polychlorinated biphenyls to aquatic humic substances: The role of substrate and sorbate properties on partitioning. Environ Sci Technol, 1999, 33: 2715-2718

14 Cornelissen G, Elmquist M, Groth I, et al. Effect of sorbate planarity on environmental black carbon sorption. Environ Sci Technol, 2004, 38: $3574-3580$

15 Qiao X L, Carmosini N, Li F, et al. Probing the primary mechanisms affecting the environmental distribution of estrogen and androgen isomers. Environ Sci Technol, 2011, 45: 3989-3995

16 Zhu D, Hyun S, Pignatello J J, et al. Evidence for $\pi-\pi$ electron donor-acceptor interactions between $\pi$-donor aromatic compounds and $\pi$-acceptor sites in soil organic matter through $\mathrm{pH}$ effects on sorption. Environ Sci Technol, 2004, 38: 4361-4368

17 Saparpakorn P, Kim J H, Hannongbua S. Investigation on the binding of polycyclic aromatic hydrocarbons with soil organic matter: A theoretical approach. Molecules, 2007, 12: 703-715

18 Niederer C, Goss K U. Quantum chemical modeling of humic acid/ air equilibrium partitioning of organic vapors. Environ Sci Technol, 2007, 41: 3646-3652

19 Frisch M J, Trucks G W, Schlegel H B, et al. Gaussian 09, Revision A.02. Gaussian, Inc., Wallingford CT, 2009

20 Kukkonen J, McCarthy J F, Oikari A. Effects of XAD-8 fractions of dissolved organic carbon on the sorption and bioavailability of organic micropollutants. Arch Environ Contam Toxicol, 1990, 19: 551-557

21 Yamamoto H, Liljestrand H M, Shimizu Y, et al. Effects of physicalchemical characteristics on the sorption of selected endocrine disruptors by dissolved organic matter surrogates. Environ Sci Technol, 2003, 37: 2646-2657

22 Perminova I V, Grechishcheva N Y, Petrosyan S V. Relationships between structure and binding affinity of humic substances for polycyclic aromatic hydrocarbons: Relevance of molecular descriptors. Environ Sci Technol, 1999, 33: 3781-3787

23 Murray J S, Lane P, Brinck T, et al. Relationships of critical constants and boiling points to computed molecular surface properties. $\mathrm{J}$ Phys Chem, 1993, 97: 9369-9373

24 Politzer P, Murray J S, Peralta-Inga Z. Molecular surface electrostatic potentials in relation to noncovalent interactions in biological systems. Int J Quantum Chem, 2001, 85: 676-684

25 Cockroft S L, Hunter C A, Lawson K R, et al. Electrostatic control of aromatic stacking interactions. J Am Chem Soc, 2005, 127: 8594-8595

Open Access This article is distributed under the terms of the Creative Commons Attribution License which permits any use, distribution, and reproduction in any medium, provided the original author(s) and source are credited. 